\title{
The incidence and prevalence of cardiovascular diseases in gout: a systematic review and meta-analysis
}

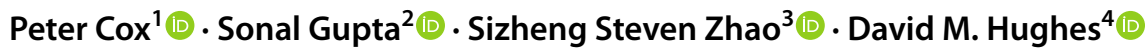

Received: 1 March 2021 / Accepted: 21 April 2021 / Published online: 13 May 2021

(c) The Author(s) 2021

\begin{abstract}
The aims of this systematic review and meta-analysis were to describe prevalence of cardiovascular disease in gout, compare these results with non-gout controls and consider whether there were differences according to geography. PubMed, Scopus and Web of Science were systematically searched for studies reporting prevalence of any cardiovascular disease in a gout population. Studies with non-representative sampling, where a cohort had been used in another study, small sample size $(<100)$ and where gout could not be distinguished from other rheumatic conditions were excluded, as were reviews, editorials and comments. Where possible meta-analysis was performed using random-effect models. Twenty-six studies comprising 949,773 gout patients were included in the review. Pooled prevalence estimates were calculated for five cardiovascular diseases: myocardial infarction $(2.8 \%$; $95 \%$ confidence interval (CI)s $1.6,5.0)$, heart failure $(8.7 \%$; 95\% CI 2.9, 23.8), venous thromboembolism $(2.1 \%$; 95\% CI 1.2, 3.4), cerebrovascular accident $(4.3 \%$; 95\% CI 1.8, 9.7) and hypertension $(63.9 \%$; $95 \%$ CI 24.5, 90.6). Sixteen studies reported comparisons with non-gout controls, illustrating an increased risk in the gout group across all cardiovascular diseases. There were no identifiable reliable patterns when analysing the results by country. Cardiovascular diseases are more prevalent in patients with gout and should prompt vigilance from clinicians to the need to assess and stratify cardiovascular risk. Future research is needed to investigate the link between gout, hyperuricaemia and increased cardiovascular risk and also to establish a more thorough picture of prevalence for less common cardiovascular diseases.
\end{abstract}

Keywords Gout $\cdot$ Cardiovascular disease $\cdot$ Prevalence $\cdot$ Meta-analysis

\section{Introduction}

Gout is an inflammatory crystal arthropathy characterised by hyperuricaemia and intra-articular monosodium urate crystal deposition. The prevalence of gout in adults has been increasing over time, with an estimated prevalence of $3.2 \%$ in the UK (5.2\% in men and $1.3 \%$ in women) [1], $3.9 \%$ in the USA (5.2\% in men and $2.7 \%$ in females) [2]

Peter Cox

hlpcox@liverpool.ac.uk

1 Institute of Systems, Molecular and Integrative Biology, University of Liverpool, Biosciences Building, Crown Street, Liverpool L69 7BE, UK

2 School of Medicine, University of Liverpool, Liverpool, UK

3 Musculoskeletal Biology, Institute of Life Course and Medical Sciences, University of Liverpool, Liverpool, UK

4 Department of Health Data Science, University of Liverpool, Liverpool, UK and $3.8 \%$ in Taiwan (5.2\% in men and $2.3 \%$ in women) [3]. It is characterised by acute attacks typically lasting up to 14 days and is associated with hyperuricaemia, purine-rich diets and increased alcohol consumption [4]. Urate is the final product of purine metabolism, a step catalysed by the xanthine oxidase enzyme making it a pharmacological target for agents such as allopurinol or febuxostat [5]. Elevated urate levels predispose to crystal precipitation in the synovial fluid. This in turn leads to recruitment of monocytes and macrophages to perform phagocytosis and release proinflammatory cytokines, resulting in a local inflammatory response causing swelling of the soft tissue and joint [5].

Both gout and subclinical hyperuricaemia are associated with adverse cardiovascular outcomes. Hyperuricaemia has been linked with an increased incidence of both coronary heart disease (CHD) and cerebrovascular accident (CVA) [6, 7]. Several studies have found gout to be associated with an increased risk of cardiovascular diseases, such as CHD and CVA, but the evidence is conflicting [8-11]. Furthermore, 
there have been no previous reviews assessing the prevalence of venous thromboembolism (VTE) in patients with gout.

The aims of this review were to: (1) describe the incidence and prevalence of cardiovascular disease in gout, (2) compare these results with non-gout controls and (3) consider whether there are differences according to geography.

\section{Methods}

The review was performed in accordance with the Preferred Reporting Items for Systematic Reviews and Meta-Analyses (PRISMA) guidelines and registered as PROSPERO CRD42021232717 [12]. In January 2021, PubMed, Scopus and Web of Science were searched using the following MeSH terms and keywords: [Gout] AND [Cardiovascular OR Cardiovascular disease].

Studies were included if they reported an adult population with gout and recorded either the number of cases of a given cardiovascular disease or the incidence of cardiovascular disease per person years. Studies with non-representative sampling (e.g. all male participants), where a cohort had been used in another study, small sample size $(<100)$ and where gout could not be distinguished from other rheumatic conditions were excluded, as were reviews, editorials and comments. There was no restriction by study setting (e.g. primary care, secondary care, outpatients) or by country. Studies reporting interventional or secondary prevention trials were excluded.

Titles and abstracts were screened for eligibility. Sample size and the prevalence of the investigated cardiovascular diseases were extracted from each study, alongside demographic data, the data source, how they defined gout and the outcome and comparisons with non-gout controls. Quality assessment was performed using a modified version of the Newcastle-Ottawa Scale. Studies were scored based on 4 aspects: representativeness (0-2), sample size (0-1), gout definition (0-1) and ascertainment of cardiovascular disease $(0-1)$. A higher score indicated better methodological quality and lower risk of bias. The review was conducted by one author (PC) and a second performed a $10 \%$ validation (SG).

Where prevalence data was reported on a cardiovascular condition in $\geq 3$ cohorts, meta-analysis was performed. Pooled prevalence was calculated using random-effect models (DerSimonian-Laird) and heterogeneity presented using the $I^{2}$ statistic. Funnel plots were produced to assess risk of publication bias. The meta-analysis was performed using $\mathrm{R}$ version 4.0.3.

\section{Results}

The search produced 6164 publications, of which 26 were included in the review after duplicates and exclusions, illustrated in Fig. 1. There was a total of 949,773 patients with gout, although one study by Singh et al. did not report the sample size of gout patients alone [13]. Eleven studies investigated populations from the USA, followed by 6 from Taiwan, 4 from the Netherlands, 3 from the UK, 1 from China and 1 from Canada.

The mean age ranged from 52.5 to 80.1 , with a medium value of 62.4, and the percentage of male participants from $60.4 \%$ to $99.1 \%$. Twelve of the studies involved participants at primary care or population level while the remaining 14 examined individuals from hospital or outpatients' settings. The study by Seminog et al. analysed 2 different cohorts comprising hospitalised patient's records: the record-linked Hospital Episode Statistics (HES) dataset for England from 1999 to 2011 and the Oxford Record Linkage Study (ORLS) dataset from 1963 to 1998 [10].

The majority of papers, $n=16$, defined gout by diagnostic code, predominantly International Classification of Diseases, with 4 accepting self-reported gout, 2 according to the Wallace Criteria, 1 by the International Classification of Primary Care (ICPC), 1 physician diagnosed, 1 by

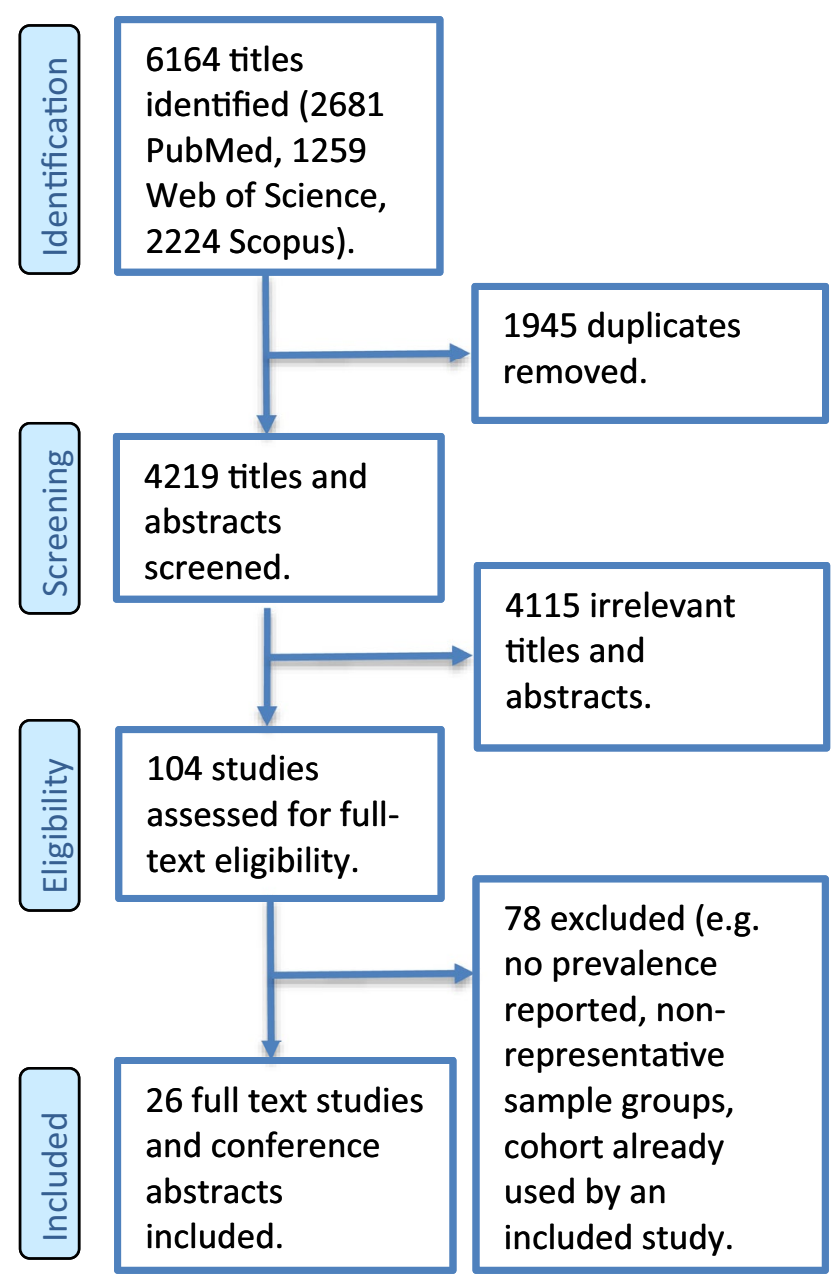

Fig. 1 Study selection flowchart 
attending rheumatic outpatients and 1 by crystal-proven joint fluid analysis. Most bias scores were 3 out of a potential 6 stars (Supplementary Table S1 and Fig S1), indicating moderate bias.

Myocardial infarction (MI) was studied in eight papers, the most frequently investigated cardiovascular disease, followed by VTE investigated in six studies. Cardiovascular disease was also predominantly defined by diagnostic code $n=18$, with 3 studies requiring a physician diagnosis, 2 by transthoracic echocardiogram and 1 each by a cardiologist reviewing resting ECGs, by the ICPC and by self-reporting The complete data extraction is provided in Supplementary Table S2.

\section{Prevalence of cardiovascular diseases}

Pooled prevalence estimates were calculated for five cardiovascular diseases, of which hypertension had the highest prevalence of $63.9 \%(24.5 \%, 90.6 \%)$ followed by heart failure with $8.7 \%(2.9 \%, 23.8 \%)$, CVA with $4.3 \%(1.8,9.7)$, MI with $2.8 \%(1.6,5.0)$ and VTE with $2.1 \%(1.2,3.4)$. The forest plots for each are shown in Fig. 2 and funnel plots provided in Supplementary Fig S2-S6. There was significant heterogeneity in the meta-analysis, with $I^{2} \geq 99 \%$ throughout.

The full results for prevalence and incidence are listed in Table 1. MI prevalence ranged from $1.3 \%$ to $19.5 \%$, with the largest study of 202,033 gout patients recording prevalence at $5.4 \%$. This was the largest study included in the review and also reported CVA prevalence at $4.9 \%$. Incidence of CVA was measured at 9.3 cases per 1000 person years and also as 7.5 in males and 13.7 in females. Heart failure prevalence ranged from $1.7 \%$ to $23.2 \%$. A small study of 647 gout patients found VTE prevalence to be $6.2 \%$ while the remaining studies were much larger and measured values between $1.0 \%$ and $2.4 \%$. The largest study investigating hypertension comprised more than 65,000 gout patients and reported prevalence of $17.8 \%$, compared with two other smaller studies which measured prevalence at above $80 \%$.

Of the remaining diseases there was a great range in reported prevalence, from $25.9 \%$ for general cardiovascular disease to $0.8 \%$ for transient ischaemic attack. Likewise, the highest incidence rate reported was that of 43.4 for atrial fibrillation, the lowest being 2.1 reported in cardiovascular mortality. There was some variation amongst rates for individual diseases, such as cardiovascular mortality incidence reported at both 23.1 and 2.1.

\section{Prevalence compared with controls}

Of the 26 studies, 16 reported comparisons with non-gout controls. These have mainly reported as adjusted hazard ratio (HR), but also as adjusted odds ratio (OR), adjusted relative risk (RR) and standardised mortality rate, with different studies measuring different outcomes such as incidence and prevalence. These results are reported in Table 2, with the general trend being that of increased risk in the gout group, particularly for MI. A small number of results indicate a risk decrease, but for each the confidence intervals cross 1.00 , so can be deemed statistically insignificant.

\section{Prevalence of cardiovascular diseases by geography}

Seven cardiovascular diseases were studied in three or more countries. The highest prevalence of MI was reported in the USA at $19.5 \%(12.6,28.0)$, followed by the UK at 5.4\% (5.3, 5.5). Other studies investigating the Netherlands, Taiwan and the USA had similar rates, including $2.0 \%(1.5,2.8), 1.7 \%$ $(1.6,1.9)$ and $1.7 \%(1.6,1.8)$ respectively. Additionally, the incidence rate per 1000 person years in the UK was higher than that of the USA and Taiwan, at 9.3 and 6.1 for males and females respectively in the UK compared with 4.1 for the USA and 2.2 for Taiwan.

The prevalence of CVA followed a similar trend with $9.5 \%(8.5,10.5)$ and $4.9 \%(4.8,5.0)$ reported in the UK compared with $1.6 \%(1.1,2.2)$ in the Netherlands. For VTE, the USA recorded the highest prevalence of $6.2 \%(4.5,8.3)$. Prevalence in the UK and Taiwan was measured at $2.4 \%$ $(2.3,2.5)$ and $1.0 \%(0.9,1.1)$ respectively. The highest incidence rate per 1000 person years was seen in the UK at 3.7, followed by Canada at 2.6 and then Taiwan measured at both 1.4 and 0.5 . The prevalence of heart failure was greatly varied measured at $23.2 \%(15.6,32.3)$ in Taiwan, both $22.8 \%$ $(21.8,23.8)$ and $5.4 \%(5.2,5.6)$ in the USA and $1.7 \%(1.1$, 2.4) in the Netherlands.

Conditions studied in 2 or less countries were not analysed according to geography.

\section{Discussion}

This systematic review and meta-analysis combined results from 26 studies and almost one million patients with gout to show that the prevalence of $\mathrm{MI}$ is $2.8 \%$, heart failure is $8.7 \%$, VTE is $2.1 \%$, CVA is $4.3 \%$ and hypertension is $63.9 \%$. The risk of cardiovascular diseases is higher in gout patients when compared with non-gout controls, as is the rate of cardiovascular mortality.

\section{Myocardial infarction}

The pooled prevalence estimate for MI of $2.8 \%$ is comparable to $3.1 \%$ in rheumatoid arthritis [35], 3.2\% in psoriatic arthritis [36] and $2.2 \%$ in axial spondyloarthritis [37]. There is an increased risk of MI compared to non-gout controls. There may be numerous explanations for this finding. 
a

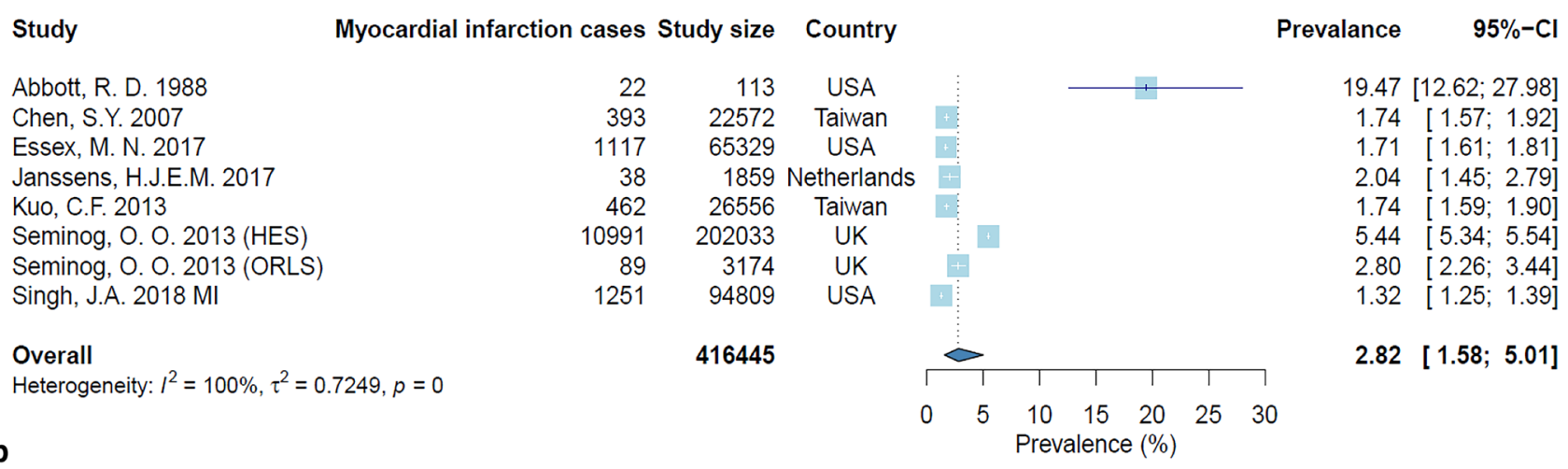

Study

Heart failure cases Study size Country

Prevalance $\quad 95 \%-\mathrm{Cl}$

Essex, M. N. 2017

Francis-Sedlak, M. 2020

Janssens, H.J.E.M. 2017

Lin, J.C. 2015

\begin{tabular}{|c|c|}
\hline 3534 & 65329 \\
\hline 1559 & 6831 \\
\hline 31 & 1859 \\
\hline 25 & 108 \\
\hline
\end{tabular}

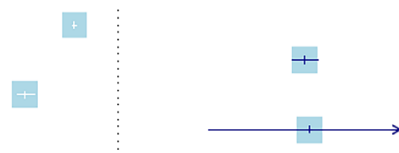

$5.41 \quad[5.24 ; 5.59]$

$22.82[21.83 ; 23.84]$

$1.67[1.14 ; 2.36]$

74127

Overall

Heterogeneity: $I^{2}=100 \%, \tau^{2}=1.4318, p=0$

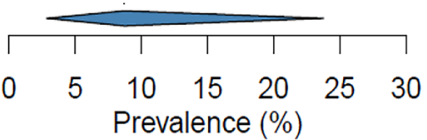

$23.15[15.57 ; 32.25]$

$8.73[2.85 ; 23.76]$

C

Study

Venous thromboembolism cases Study size Country

Prevalance $\quad 95 \%-\mathrm{Cl}$

Chiu, C. C. 2016

Kubota, Y. 2016

Li, L. 2020

Raisch, D.W. 2009 ABSTRACT

Sultan, A. A. 2019

367
40
2065
2540
1481

\begin{tabular}{rc|}
35959 & Taiwan \\
647 & USA \\
130708 & Canada \\
156809 & USA \\
62234 & UK
\end{tabular}

$1.02[0.92 ; 1.13]$

$6.18[4.45 ; 8.32]$

$1.58[1.51 ; 1.65]$

$1.62[1.56 ; 1.68]$

$2.38[2.26 ; 2.50]$

386357

Overall

Heterogeneity: $I^{2}=99 \%, \tau^{2}=0.3576, p<0.01$

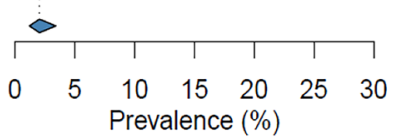

$2.05[1.22 ; 3.43]$

d

Study

Janssens, H.J.E.M. 2017

Seminog, O. O. 2013 (HES)

Seminog, O. O. 2013 (ORLS)

Overall

Heterogeneity: $I^{2}=99 \%, \tau^{2}=0.5847, p<0.01$

e

Study

Hypertension cases Study size Country

$\begin{array}{rrr}29 & 1859 & \text { Netherlands } \\ 9960 & 202033 & \text { UK } \\ 300 & 3174 & \text { UK } \\ & & \\ & & \end{array}$

207066

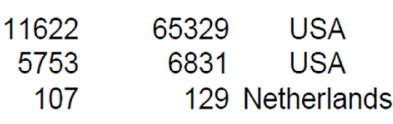

72289

Overall

Heterogeneity: $I^{2}=100 \%, \tau^{2}=2.2321, p=0$
Essex, M. N. 2017

Francis-Sedlak, M. 2020

Meek, I.L. 2013

\section{Prevalance $\quad 95 \%-\mathrm{Cl}$}

$1.56[1.05 ; 2.23]$

$4.93[4.84 ; 5.03]$

$9.45[8.46 ; 10.52]$

$4.27[1.83 ; 9.67]$

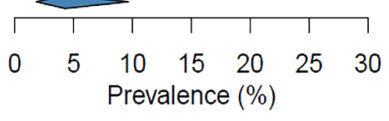

Prevalance $\quad 95 \%-\mathrm{Cl}$

17.79 [17.50; 18.09]

84.22 [83.33; 85.08]

82.95 [75.33; 88.99]

63.94 [24.51; 90.64]

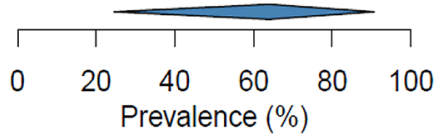

Fig. 2 Forest plots of pooled prevalence of: a $2.8 \%$ for myocardial infarction, b $8.7 \%$ for heart failure, $\mathbf{c} 2.1 \%$ for venous thromboembolism, d $4.3 \%$ for cerebrovascular accident and e $63.9 \%$ for hypertension 
Table 1 Summary of studies included in the systematic review

\begin{tabular}{|c|c|c|c|c|c|}
\hline Study & Country & Source & Sample Size & Prevalence & $\begin{array}{l}\text { Incidence } \\
\text { per person } \\
1000 \text { years }\end{array}$ \\
\hline \multicolumn{6}{|l|}{ Myocardial infarction } \\
\hline Abbott et al. [8] & USA & Framingham Study & 113 & 19.47 & \\
\hline Chen et al. [14] & Taiwan & Ho-Ping Gout Database, inpatients & 22,572 & 1.74 & \\
\hline Essex et al. [15] & USA & Humedica database & 65,329 & 1.71 & \\
\hline Janssens et al. [16] & Netherlands & GP Database & 1859 & 2.04 & \\
\hline Kuo et al. [3] & Taiwan & Taiwanese National Health Insurance database & 26,556 & 1.74 & 2.2 \\
\hline Seminog et al. (HES) [10] & UK & Hospital Episode Statistics for England & 202,033 & 5.44 & \\
\hline Seminog et al. (ORLS) [10] & UK & Oxford Record Linkage Study & 3174 & 2.8 & \\
\hline Singh et al. MI [17] & USA & Medicare $5 \%$ claims data & 94,809 & 1.32 & 4.1 \\
\hline Clarson et al. [18] & UK & Clinical Practice Research Datalink & 8386 & & M 9.27, F 6.11 \\
\hline \multicolumn{6}{|l|}{ Cerebrovascular accident } \\
\hline Janssens et al. [16] & Netherlands & GP Database & 1859 & 1.56 & \\
\hline Seminog et al. (HES) [10] & UK & Hospital Episode Statistics for England & 202,033 & 4.93 & \\
\hline Seminog et al. (ORLS) [10] & UK & Oxford Record Linkage Study & 3174 & 9.45 & \\
\hline Clarson et al. [18] & UK & Clinical Practice Research Datalink & 8386 & & M 7.45, F 13.71 \\
\hline Colantonio et al. [11] & USA & REGARDS cohort & 187 & & 9.3 \\
\hline \multicolumn{6}{|l|}{ Heart failure } \\
\hline Essex et al. [15] & USA & Humedica database & 65,329 & 5.41 & \\
\hline Francis-Sedlak et al. [19] & USA & Humanan Research Database & 6831 & 22.82 & \\
\hline Janssens et al. [16] & Netherlands & GP Database & 1859 & 1.67 & \\
\hline Lin et al. [20] & Taiwan & N/A & 108 & 23.15 & \\
\hline Colantonio et al. [11] & USA & REGARDS cohort & 187 & & 13.1 \\
\hline \multicolumn{6}{|l|}{ Venous thromboembolism } \\
\hline Chiu et al. [21] & Taiwan & Taiwanese National Health Insurance database & 35,959 & 1.02 & 1.348 \\
\hline Kubota et al. [22] & USA & Atherosclerosis Risk in Communities Study & 647 & 6.18 & \\
\hline Raisch et al. ABSTRACT [23] & USA & Veterans Affairs national database & 156,809 & 1.62 & \\
\hline Li et al. [24] & Canada & Population Data BC Database & 130,708 & 1.58 & 2.63 \\
\hline Sultan et al. [25] & UK & Primary Care and NHS Hospital databases & 62,234 & 2.38 & 3.73 \\
\hline Huang et al. [26] & Taiwan & National Health Insurance Research database & 57,981 & & 0.526 \\
\hline \multicolumn{6}{|l|}{ Cardiovascular mortality } \\
\hline Disveld et al. [27] & Netherlands & Gout Arnhem-Liemers Cohort & 700 & & 7.71 \\
\hline Kuo et al. [28] & Taiwan & $\begin{array}{l}\text { Health screening programme in Chang Gung } \\
\text { Memorial Hospital }\end{array}$ & 1311 & & 2.09 \\
\hline Stack et al. [29] & USA & $\begin{array}{l}\text { Third National Health and Nutrition Examination } \\
\text { Survey }\end{array}$ & 468 & & 23.1 \\
\hline \multicolumn{6}{|l|}{ Hypertension } \\
\hline Essex et al. [15] & USA & Humedica database & 65,329 & 17.79 & \\
\hline Francis-Sedlak et al. [19] & USA & Humanan Research Database & 6831 & 84.22 & \\
\hline Meek et al. [30] & Netherlands & $\begin{array}{l}\text { Arthritis Center Twente (outpatients) and the } \\
\text { Doetinchem Cohort }\end{array}$ & 129 & 83 & \\
\hline Pan et al. [31] & China & Singapore Chinese Health Study & 573 & & 52.54 \\
\hline \multicolumn{6}{|l|}{ Angina } \\
\hline Abbott et al. [8] & USA & Framingham Study & 113 & 20.35 & \\
\hline Janssens et al. [16] & Netherlands & GP Database & 1859 & 0.97 & \\
\hline Clarson et al. [18] & UK & Clinical Practice Research Datalink & 8386 & & M 11.80, F 12.32 \\
\hline \multicolumn{6}{|l|}{ Transient ischaemic attack } \\
\hline Janssens et al. [16] & Netherlands & GP Database & 1859 & 0.81 & \\
\hline Clarson et al. [18] & UK & Clinical Practice Research Datalink & 8386 & & M 6.04, F 9.34 \\
\hline
\end{tabular}


Table 1 (continued)

\begin{tabular}{|c|c|c|c|c|c|}
\hline Study & Country & Source & Sample Size & Prevalence & $\begin{array}{l}\text { Incidence } \\
\text { per person } \\
1000 \text { years }\end{array}$ \\
\hline \multicolumn{6}{|l|}{ Atrial fibrillation } \\
\hline Francis-Sedlak et al. [19] & USA & Humanan Research Database & 6831 & 21.48 & \\
\hline Kim et al. [32] & USA & United Health Care database & 70,015 & 1.47 & 7.19 \\
\hline Singh et al. AF [13] & USA & Medicare $5 \%$ claims data & N/A & & 43.4 \\
\hline \multicolumn{6}{|l|}{ Peripheral vascular disease } \\
\hline Janssens et al. [16] & Netherlands & GP Database & 1859 & 1.51 & \\
\hline Clarson et al. [18] & UK & Clinical Practice Research Datalink & 8386 & & M 5.60, F 7.09 \\
\hline \multicolumn{6}{|l|}{ Any coronary heart disease } \\
\hline Clarson et al. [18] & UK & Clinical Practice Research Datalink & 8386 & & M 28.46, F 9.11 \\
\hline Colantonio et al. [11] & USA & REGARDS cohort & 187 & & 16.0 \\
\hline \multicolumn{6}{|l|}{ Aortic stenosis } \\
\hline Chang et al. [33] & USA & Outpatients & 112 & 21.43 & \\
\hline \multicolumn{6}{|l|}{ Aortic aneurysm } \\
\hline Janssens et al. [16] & Netherlands & GP Database & 1859 & 0.91 & \\
\hline \multicolumn{6}{|l|}{ Cardiovascular disease } \\
\hline Janssens et al. [34] & Netherlands & Continuous Morbidity Registration & 170 & 25.88 & \\
\hline
\end{tabular}

F Female, HES Hospital Episode Statistics, M Male, ORLS Oxford Record Linkage Study

Classical cardiovascular risk factors such as obesity, diabetes mellitus and hypertension have been shown to be more prevalent among those with gout [35]. There remains an association between gout and cardiovascular disease after adjusting for these factors, illustrating that gout conveys its own independent risk, potentially as a result of intermittent and chronic inflammation [38]. Even in patients without traditional risk factors, the risk of MI was found to be high, leading to the suggestion that gout is an early manifestation of metabolic abnormalities [3]. It has also been suggested that peripheral joint inflammation produces a greater systemic inflammatory response which may contribute to the increased prevalence of cardiovascular conditions [36]. While the underlying pathological mechanism remains unclear, this data indicates the effect of gout on cardiovascular disease has been underestimated for some time and needs reconsideration.

\section{Heart failure}

The pooled prevalence value for heart failure of $8.7 \%$ was produced from results from individual studies which ranged from $1.7 \%$ to $23.2 \%$, reflected in the broad $95 \%$ confidence interval of 2.9-23.8. These varying results may be attributable to differences in methodology, for example the prospective study by Lin et al. which investigated patients who both had gout and had undergone a transthoracic echocardiogram [20]. As this is not a routine investigation for patients with gout, it may well have increased the likelihood of this population having left ventricular dysfunction as the study reported. Furthermore, gout is predominantly managed in primary care. This means this cohort potentially suffered from more severe gout, or were more complex patients who required secondary care, both of which may increase the chances of comorbidities being present. The other study to report a large prevalence was by Francis-Sedlak et al., potentially stemming from a strict eligibility criteria which included just 6831 of 539,802 identified gout patients [19]. Enrolment in the database 6 months before and after diagnosis, 90 days of continuous urate lowering therapy and 2 subsequent serum uric acid measurements were required for inclusion. Comparable figures for other rheumatic conditions include $1.6 \%$ for rheumatoid arthritis [39], 1.3\% for psoriatic arthritis [36], and 1.8 for axial spondyloarthritis [37] which may be closer to the true value.

The same argument of limited study groups and broad confidence intervals could also be made for the pooled prevalence for CVA of $4.3 \%(1.8 \%, 9.7 \%)$ and hypertension $63.9 \%(24.5,90.6)$. The inability to determine a more precise estimate of prevalence may reflect a greater failing by the medical community to investigate this association, particularly given the potentially fatal nature of stroke.

\section{Venous thromboembolism}

This is the first meta-analysis of VTE prevalence in gout populations which found a pooled prevalence of $2.1 \%$, with most studies reporting increased risk compared with nongout controls. VTE has also been shown to have an increased risk in other types of inflammatory arthritis [40, 41]. This 
Table 2 Studies comparing results between gout patients and non-gout controls

\begin{tabular}{|c|c|c|c|c|}
\hline Cardiovascular disease & Study & Measure of effect & Effect size & $95 \%$ confidence interval \\
\hline \multirow[t]{5}{*}{ Myocardial infarction } & Clarson et al. [18] & $\begin{array}{l}\text { HR for MI risk adjusted for age, sex, } \\
\text { BMI, smoking status, alcohol, Charl- } \\
\text { son comorbidity index, comorbidities } \\
\text { and medication }\end{array}$ & M 1.12, F 0.97 & M $1.00-1.27$, F $0.77-1.22$ \\
\hline & Seminog et al. (HES) [10] & $\begin{array}{l}\text { RR for MI adjusted for age, sex, time, } \\
\text { area of residence and deprivation }\end{array}$ & 1.82 & $1.78-1.85$ \\
\hline & Seminog et al. (ORLS) [10] & $\begin{array}{l}\mathrm{RR} \text { for MI adjusted for age, sex, time, } \\
\text { area of residence and deprivation }\end{array}$ & 1.95 & $1.57-2.40$ \\
\hline & Kuo et al. [3] & $\begin{array}{l}\text { HR for incidence adjusted for age, sex } \\
\text { and comorbidities }\end{array}$ & 1.23 & $1.11-1.36$ \\
\hline & Singh et al. MI [17] & $\begin{array}{l}\text { HR for incidence adjusted for age, sex, } \\
\text { comorbidities and medication }\end{array}$ & 2.08 & $1.95-2.21$ \\
\hline \multirow[t]{4}{*}{ Cerebrovascular accident } & Clarson et al. [18] & $\begin{array}{l}\text { HR for CVA risk adjusted for age, sex, } \\
\text { BMI, smoking status, alcohol, Charl- } \\
\text { son comorbidity index, comorbidities } \\
\text { and medication }\end{array}$ & M 0.93, F 1.34 & M 0.81-1.06, F 1.15-1.57 \\
\hline & Seminog et al. (HES) [10] & $\begin{array}{l}\text { RR for CVA adjusted for age, sex, time, } \\
\text { area of residence and deprivation }\end{array}$ & 1.71 & $1.68-1.75$ \\
\hline & Seminog et al. (ORLS) [10] & $\begin{array}{l}\text { RR for CVA adjusted for age, sex, time, } \\
\text { area of residence and deprivation }\end{array}$ & 1.91 & $1.70-2.14$ \\
\hline & Colantonio et al. [11] & $\begin{array}{l}\text { HR for prevalence adjusted for age, sex, } \\
\text { race, region of residence, income, } \\
\text { education, alcohol, smoking, BMI, } \\
\text { physical activity, dietary patterns, } \\
\text { comorbidities and medication }\end{array}$ & 0.83 & $0.48-1.43$ \\
\hline \multirow[t]{5}{*}{ Venous thromboembolism } & Chiu et al. [21] & HR for DVT risk & 1.38 & $1.18-1.62$ \\
\hline & Sultan et al. [25] & $\begin{array}{l}\text { HR for VTE risk adjusted for age, sex, } \\
\text { BMI, alcohol, smoking, time, depriva- } \\
\text { tion, hospital admission and medica- } \\
\text { tion }\end{array}$ & 1.25 & $1.15-1.35$ \\
\hline & Kubota et al. [22] & $\begin{array}{l}\text { HR for VTE risk adjusted for age, sex, } \\
\text { race, BMI, smoking and comorbidity }\end{array}$ & 1.33 & $0.95-1.86$ \\
\hline & Huang et al. [26] & $\begin{array}{l}\text { HR for incidence adjusted for age, sex } \\
\text { and comorbidities }\end{array}$ & 1.66 & $1.37-2.01$ \\
\hline & Li et al. [24] & $\begin{array}{l}\text { HR for incidence adjusted for age, } \\
\text { sex, healthcare utilisation, Charlson } \\
\text { comorbidities index, comorbidities and } \\
\text { medications }\end{array}$ & 1.22 & $1.13-1.32$ \\
\hline Heart failure & Colantonio et al. [11] & $\begin{array}{l}\text { HR for prevalence adjusted for age, sex, } \\
\text { race, region of residence, income, } \\
\text { education, alcohol, smoking, BMI, } \\
\text { physical activity, dietary patterns, } \\
\text { comorbidities and medication }\end{array}$ & 1.97 & $1.22-3.19$ \\
\hline \multirow[t]{2}{*}{ Cardiovascular mortality } & Stack et al. [29] & $\begin{array}{l}\text { HR for prevalence adjusted for age, sex, } \\
\text { race, BMI and comorbidities }\end{array}$ & 1.58 & $1.13-2.19$ \\
\hline & Disveld et al. [27] & Standardized mortality rate & 6.75 & $4.64-8.86$ \\
\hline \multirow[t]{2}{*}{ Atrial fibrillation } & Kim et al. [32] & $\begin{array}{l}\text { HR for incidence adjusted age, sex, } \\
\text { comorbidities, medication and health- } \\
\text { care utilisation }\end{array}$ & 1.21 & $1.11-1.33$ \\
\hline & Singh et al. AF [13] & $\begin{array}{l}\text { HR for incidence adjusted for age, sex } \\
\text { and medication }\end{array}$ & 1.92 & $1.88-1.96$ \\
\hline Angina & Clarson et al. [18] & $\begin{array}{l}\text { HR for angina risk adjusted for age, sex, } \\
\text { BMI, smoking status, alcohol, charlson } \\
\text { comorbidity index, comorbidities and } \\
\text { medication }\end{array}$ & M 1.02, F 1.28 & M $0.92-1.13$, F $1.09-1.51$ \\
\hline
\end{tabular}


Table 2 (continued)

\begin{tabular}{|c|c|c|c|c|}
\hline Cardiovascular disease & Study & Measure of effect & Effect size & $95 \%$ confidence interval \\
\hline Transient ischaemic attack & Clarson et al. [18] & $\begin{array}{l}\text { HR for transient ischaemic attack risk } \\
\text { adjusted for age, sex, BMI, smoking } \\
\text { status, alcohol, charlson comorbidity } \\
\text { index, comorbidities and medication }\end{array}$ & M 1.02, F 1.26 & M $0.88-1.18$, F $1.05-1.53$ \\
\hline Peripheral vascular disease & Clarson et al. [18] & $\begin{array}{l}\text { HR for peripheral vascular disease risk } \\
\text { adjusted for age, sex, BMI, smoking } \\
\text { status, alcohol, charlson comorbidity } \\
\text { index, comorbidities and medication }\end{array}$ & M 1.18, F 1.89 & M $1.01-1.38$, F $1.50-2.38$ \\
\hline \multirow[t]{2}{*}{ Hypertension } & Pan et al. [31] & $\begin{array}{l}\text { HR for hypertension risk adjusted } \\
\text { for age, sex, year, education, BMI, } \\
\text { alcohol, smoking, physical activity and } \\
\text { diabetes }\end{array}$ & 1.18 & $1.02-1.37$ \\
\hline & Meek et al. 2013 [30] & $\begin{array}{l}\text { OR for prevalence adjusted for age and } \\
\text { sex }\end{array}$ & 2.7 & $1.7-4.3$ \\
\hline \multirow[t]{2}{*}{ Any coronary heart disease } & Clarson et al. [18] & $\begin{array}{l}\text { HR for coronary heart disease risk } \\
\text { adjusted for age, sex, BMI, smoking } \\
\text { status, alcohol, charlson comorbidity } \\
\text { index, comorbidities and medication }\end{array}$ & M 1.08, F 1.25 & M 1.01-1.15, F 1.12-1.39 \\
\hline & Colantonio et al. [11] & $\begin{array}{l}\text { HR for prevalence adjusted for age, sex, } \\
\text { race, region of residence, income, } \\
\text { education, alcohol, smoking, BMI, } \\
\text { physical activity, dietary patterns, } \\
\text { comorbidities and medication }\end{array}$ & 1.21 & $0.79-1.84$ \\
\hline Aortic stenosis & Chang et al. [33] & OR for prevalence matched for age & 2.08 & $1.00-4.32$ \\
\hline
\end{tabular}

Bold text indicates statistical significance

BMI Body mass index, DVT Deep vein thrombosis, HES Hospital Episode Statistics, HR Hazard ratio, OR Odds ratio, ORLS Oxford Record Linkage Study, $R R$ Relative risk

predisposition to coagulation may be the result of inflammatory damage to the vascular endothelium [24]. It has been proposed that activation of the nucleotide-binding domain, leucine-rich-containing family, pyrin domain-containing-3 (NLRP3) inflammasome, which in turn stimulates release of interleukin-1 $\beta$, could enhance this inflammatory response [42]. An idea to resolve this could be the long-term management of gout involving a serum urate target. This is a controversial topic, with questions over the number needed to treat and what the impacts, if any, of a long-term lowered serum urate would be [42].

\section{Cardiovascular mortality}

All studies found the risk of cardiovascular mortality to be increased when compared with non-gout controls. A study by Kok et al. highlighted a finding that gout conferred a protective effect on cardiovascular mortality in those with chronic kidney disease [43]. In explaining this finding, the question is raised that perhaps it is not gout but rather uratelowering therapies such as allopurinol that is the source of the reduction in cardiovascular risk. In hyperuricaemic patients, allopurinol has been associated with a reduced rate of all-cause mortality (HR 0.78; 95\% CI 0.67, 0.91) [44], and of major cardiovascular events (HR 0.89; 95\% CI 0.81 ,
0.97) [45]. A small-scale prospective randomized trial of patients with chronic kidney disease found that allopurinol compared to treatment as usual reduced both the risk of cardiovascular events and of hospitalisation [46]. However, in contrast to this a cohort study from Taiwan in a gout population did not observe any beneficial effect from allopurinol on cardiovascular risk [47], indicating the need for further research into the cardiovascular effect of allopurinol on patients with gout.

\section{Hypertension}

Hypertension pooled prevalence was calculated at $63.9 \%$, a finding which merits careful thought as hypertension is an important risk factor for the majority of the cardiovascular diseases mentioned in this review. This finding is derived from just 3 studies and may demonstrate a lack of quality evidence in this broader aspect of gout management. Evaluating these studies individually, the study by Francis-Sedlak et al. employed a strict inclusion criteria as previously mentioned, while the study by Meek et al. investigated a small sample size from a rheumatology outpatients department which implemented routine cardiovascular screening, potentially increasing the chance of detecting hypertension $[19,30]$. The results of these studies vary considerably 
compared to that of Essex et al., which examined a much larger gout cohort at a population level [15]. Previously, uric acid has been shown to stimulate vascular smooth muscle cell proliferation in vitro, as well as both angiotensinogen and angiotensin II production [48]. A link has also been detected between hyperuricaemia and hypertension in animal models, noting elevated renin expression suggesting the underlying mechanism involves the renin-angiotensin system [49]. These studies provide plausibility for our finding of a very high hypertension prevalence in gout patients. Beyond MI, CVA and VTE there have been few studies looking at each condition and on occasion they report conflicting findings. This has thus far made it difficult to draw a conclusive impression on the effect of gout on cardiovascular disease prevalence for some of the less common conditions.

\section{Prevalence by geography}

Another aim of this review was to look for any differences relative to geography. Looking at the seven cardiovascular conditions studied in 3 or more countries, the highest prevalence of MI was recorded in the USA as $19.5 \%$. This figure appears unusually high and may be the result of a relatively small sample size of 113 . This result aside, there appears to be a marked increase in prevalence of $\mathrm{MI}$ in the UK, recorded at $5.4 \%$ and $2.8 \%$, with the other studies investigating Taiwan, the Netherlands and the USA all having similar smaller rates in the range $1.3 \%$ to $2.0 \%$. This trend was repeated for CVA, with prevalence of $4.9 \%$ and $9.5 \%$ reported in the UK compared with $1.6 \%$ in the Netherlands.

It was difficult to identify any reliable patterns when analysing the results by country. This may be down to difference in study design and methodology which in turn impacts what measurements are recorded and how that data is reported. The result is that not all the data for each cardiovascular disease is comparable to each other. This coupled with the general lack of studies present within the literature means interpreting trends between countries is challenging.

\section{Clinical implications}

Previous efforts to estimate cardiovascular risk in gout patients have found that when stratified using a risk assessment tool, $56.3 \%$ had their cardiovascular risk upgraded after undergoing a carotid ultrasound to assess for the presence of atheromatous plaques [50]. Another study found that after adding gout as a risk factor for cardiovascular events to the risk assessment tool, $38.3 \%$ of patients had their risk upgraded [51]. New classification tools may be required to better evaluate the cardiovascular implications of gout. Additionally, raised awareness could allow for more screening for the risk factors of increased prevalence, such as diabetes and hypertension. This intervention could be promoted alongside several other rheumatic conditions, such as psoriatic arthritis or axial spondyloarthritis, to nurture the understanding that the association between rheumatic conditions and cardiovascular diseases exists beyond that of just the well-established link with rheumatoid arthritis.

Furthermore, these results are in line with other studies which have shown an increased risk for sufferers of hyperuricaemia for both MI and CVA $[6,7,52]$. With the current understanding of the underlying process limited, whether synergistic or exclusive, it appears gout and hyperuricaemia have a detrimental effect on the cardiovascular system. Several studies have made an association between serum uric acid and metabolic syndrome [53, 54], suggesting it may have homogenous actions, such as activation of the sympathetic nervous system, renin-angiotensin system and increased levels of pro-inflammatory adipokines and cytokines, which confer an elevated cardiovascular risk through factors including raised heart rate, circulating blood volume and vascular resistance [55].

\section{Limitations}

A strength of this review is the broad inclusion of cardiovascular diseases. There do not appear to be any other reviews that have cast their net as wide when examining the cardiovascular impact of gout. While this has resulted in some sparse reporting of some conditions, for example in aortic stenosis, it lays groundwork for future studies to investigate these trends further.

Limitations within the review include the lack of studies, particularly with large sample sizes, which reported prevalence of given cardiovascular diseases in gout populations. This led to some imprecision in the results which manifested as large confidence intervals in the meta-analysis. It is also plausible that patients with gout would visit healthcare professionals more often than a non-gout control. This would present more opportunity to screen for and diagnose cardiovascular disease. This may result in an overestimation of prevalence and explain why nearly all studies found an increased prevalence when compared to non-gout controls [36]. The high heterogeneity seen in the meta-analysis could be due to different types of studies being carried out in different settings, leading to more uncertainty in the pooled prevalence estimates.

\section{Conclusion}

In summary, this systematic review and meta-analysis highlights the increased prevalence of numerous cardiovascular diseases amongst patients with gout. These results do well to establish a pooled prevalence for several conditions, particularly MI and VTE. This draws attention to the challenge for clinicians to be more vigilant of an increased cardiovascular 
burden in gout patients. Future research is needed to investigate the link between gout, hyperuricaemia and increased cardiovascular risk and also to establish a more thorough picture of prevalence for the wide variety of cardiovascular diseases.

Supplementary Information The online version contains supplementary material available at https://doi.org/10.1007/s00296-021-04876-6.

\section{Declarations}

Conflict of interest The authors have no conflicts of interest or funding to declare.

Open Access This article is licensed under a Creative Commons Attribution 4.0 International License, which permits use, sharing, adaptation, distribution and reproduction in any medium or format, as long as you give appropriate credit to the original author(s) and the source, provide a link to the Creative Commons licence, and indicate if changes were made. The images or other third party material in this article are included in the article's Creative Commons licence, unless indicated otherwise in a credit line to the material. If material is not included in the article's Creative Commons licence and your intended use is not permitted by statutory regulation or exceeds the permitted use, you will need to obtain permission directly from the copyright holder. To view a copy of this licence, visit http://creativecommons.org/licenses/by/4.0/.

\section{References}

1. Kuo C-F, Grainge MJ, Mallen C, Zhang W, Doherty M (2015) Rising burden of gout in the UK but continuing suboptimal management: a nationwide population study. Ann Rheum Dis 74(4):661-667

2. Singh G, Lingala B, Mithal A (2019) Gout and hyperuricaemia in the USA: prevalence and trends. Rheumatology 58(12):2177-2180

3. Kuo C-F, Yu K-H, See L-C, Chou I-J, Ko Y-S, Chang H-C, Chiou M-J, Luo S-F (2013) Risk of myocardial infarction among patients with gout: a nationwide population-based study. Rheumatology 52(1):111-117

4. Singh JA, Reddy SG, Kundukulam J (2011) Risk factors for gout and prevention: a systematic review of the literature. Curr Opin Rheumatol 23(2):192

5. Choi HK, Mount DB, Reginato AM (2005) Pathogenesis of gout. Ann Intern Med 143(7):499-516

6. Kim SY, Guevara JP, Kim KM, Choi HK, Heitjan DF, Albert DA (2010) Hyperuricemia and coronary heart disease: a systematic review and meta-analysis. Arthritis Care Res Off J Am Coll Rheumatol 62(2):170-180

7. Kim SY, Guevara JP, Kim KM, Choi HK, Heitjan DF, Albert DA (2009) Hyperuricemia and risk of stroke: a systematic review and meta-analysis. Arthritis Care Res 61(7):885-892

8. Abbott RD, Brand FN, Kannel WB, Castelli WP (1988) Gout and coronary heart disease: the Framingham Study. J Clin Epidemiol 41(3):237-242

9. Gelber AC, Klag MJ, Mead LA, Thomas J, Thomas DJ, Pearson TA, Hochberg MC (1997) Gout and risk for subsequent coronary heart disease: the Meharry-Hopkins study. Arch Intern Med 157(13):1436-1440

10. Seminog OO, Goldacre MJ (2013) Gout as a risk factor for myocardial infarction and stroke in England: evidence from record linkage studies. Rheumatology 52(12):2251-2259
11. Colantonio LD, Saag KG, Singh JA, Chen L, Reynolds RJ, Gaffo A, Plante TB, Curtis JR, Bridges SL, Levitan EB (2020) Gout is associated with an increased risk for incident heart failure among older adults: the REasons for geographic and racial differences in stroke (REGARDS) cohort study. Arthritis Res Ther 22:1-13

12. Moher D, Liberati A, Tetzlaff J, Altman DG, Group P (2009) Preferred reporting items for systematic reviews and meta-analyses: the PRISMA statement. PLoS Med 6(7):e1000097

13. Singh JA, Cleveland JD (2018) Gout and the risk of incident atrial fibrillation in older adults: a study of US Medicare data. RMD Open 4(2):e000712

14. Chen S-Y, Chen C-L, Shen M-L (2007) Severity of gouty arthritis is associated with Q-wave myocardial infarction: a large-scale, cross-sectional study. Clin Rheumatol 26(3):308-313

15. Essex MN, Hopps M, Bienen EJ, Udall M, Mardekian J, Makinson GT (2017) Evaluation of the relationship between serum uric acid levels and cardiovascular events in patients with gout: a retrospective analysis using electronic medical record data. JCR J Clin Rheumatol 23(3):160-166

16. Janssens HJ, Arts PG, Schalk BW, Biermans MC (2017) Gout and rheumatoid arthritis, both to keep in mind in cardiovascular risk management: a primary care retrospective cohort study. Jt Bone Spine 84(1):59-64

17. Singh JA, Cleveland JD (2018) Gout and the risk of myocardial infarction in older adults: a study of Medicare recipients. Arthritis Res Ther 20(1):109

18. Clarson LE, Hider SL, Belcher J, Heneghan C, Roddy E, Mallen CD (2015) Increased risk of vascular disease associated with gout: a retrospective, matched cohort study in the UK clinical practice research datalink. Ann Rheum Dis 74(4):642-647

19. Francis-Sedlak M, LaMoreaux B, Padnick-Silver L, Holt RJ, Bello AE (2020) Characteristics, comorbidities, and potential consequences of uncontrolled gout: an insurance-claims database study. Rheumatol Ther 8:1-15

20. Lin J-C, Lin C-L, Chen M-C, Chang P-J, Chang S-T, Chung C-M, Pan K-L (2015) Gout, not hyperuricemia alone, impairs left ventricular diastolic function. Arthritis Res Ther 17(1):323

21. Chiu C-C, Chen Y-T, Hsu C-Y, Chang C-C, Huang C-C, Leu H-B, Li S-Y, Kuo S-C, Huang P-H, Chen J-W (2016) Association between previous history of gout attack and risk of deep vein thrombosis-a nationwide population-based cohort study. Sci Rep $6(1): 1-7$

22. Kubota Y, McAdams-DeMarco M, Folsom AR (2016) Serum uric acid, gout, and venous thromboembolism: the atherosclerosis risk in communities study. Thromb Res 144:144-148

23. Raisch D, Rice J, Campbell H (2009) PMS1 risk assessment of cardiovascular thrombotic events, other cardiac events, and mortality among veterans affairs patients with difficult to manage gout. Value Health 12(7):A433

24. Li L, McCormick N, Sayre EC, Esdaile JM, Lacaille D, Xie H, Choi HK, Aviña-Zubieta JA (2020) Trends of venous thromboembolism risk before and after diagnosis of gout: a general population-based study. Rheumatology 59(5):1099-1107

25. Sultan AA, Muller S, Whittle R, Roddy E, Mallen C, Clarson L (2019) Venous thromboembolism in patients with gout and the impact of hospital admission, disease duration and urate-lowering therapy. CMAJ 191(22):E597-E603

26. Huang C-C, Huang P-H, Chen J-H, Lan J-L, Tsay GJ, Lin H-Y, Tseng C-H, Lin C-L, Hsu C-Y (2015) An independent risk of gout on the development of deep vein thrombosis and pulmonary embolism: a nationwide, population-based cohort study. Medicine 94(51):e2140

27. Disveld IJ, Zoakman S, Tim LTA, Rongen GA, Kienhorst LB, Janssens HJ, Fransen J, Janssen M (2019) Crystal-proven gout patients have an increased mortality due to cardiovascular diseases, cancer, and infectious diseases especially when having 
tophi and/or high serum uric acid levels: a prospective cohort study. Clin Rheumatol 38(5):1385-1391

28. Kuo C-F, See L-C, Luo S-F, Ko Y-S, Lin Y-S, Hwang J-S, Lin C-M, Chen H-W, Yu K-H (2010) Gout: an independent risk factor for all-cause and cardiovascular mortality. Rheumatology 49(1):141-146

29. Stack A, Hanley A, Casserly L, Cronin C, Abdalla A, Kiernan T, Murthy B, Hegarty A, Hannigan A, Nguyen H (2013) Independent and conjoint associations of gout and hyperuricaemia with total and cardiovascular mortality. QJM Int J Med 106(7):647-658

30. Meek IL, Picavet HSJ, Vonkeman HE, Verschuren WM, Van De Laar MA (2013) Increased cardiovascular risk factors in different rheumatic diseases compared with the general population. Rheumatology 52(1):210-216

31. Pan A, Teng GG, Yuan J-M, Koh W-P (2015) Bidirectional association between self-reported hypertension and gout: The Singapore Chinese Health Study. PLoS ONE 10(10):e0141749

32. Kim SC, Liu J, Solomon DH (2016) Risk of incident atrial fibrillation in gout: a cohort study. Ann Rheum Dis 75(8):1473-1478

33. Chang K, Yokose C, Tenner C, Oh C, Donnino R, Choy-Shan A, Pike VC, Shah BD, Lorin JD, Krasnokutsky S (2017) Association between gout and aortic stenosis. Am J Med 130(2):230.e231-230. e238

34. Janssens H, Van de Lisdonk E, Bor H, Van den Hoogen H, Janssen M (2003) Gout, just a nasty event or a cardiovascular signal? A study from primary care. Fam Pract 20(4):413-416

35. Zhu Y, Pandya BJ, Choi HK (2012) Comorbidities of gout and hyperuricemia in the US general population: NHANES $2007-$ 2008. Am J med 125(7):679-687.e671

36. Gupta S, Syrimi Z, Hughes DM, Zhao SS (2021) Comorbidities in psoriatic arthritis: a systematic review and meta-analysis. Rheumatol Int 125:1-10

37. Zhao SS, Robertson S, Reich T, Harrison NL, Moots RJ, Goodson NJ (2020) Prevalence and impact of comorbidities in axial spondyloarthritis: systematic review and meta-analysis. Rheumatology 59(Supplement_4):iv47-iv57

38. Choi HK, Curhan G (2007) Independent impact of gout on mortality and risk for coronary heart disease. Circulation 116(8):894-900

39. Nikiphorou E, de Lusignan S, Mallen CD, Khavandi K, Bedarida G, Buckley CD, Galloway J, Raza K (2020) Cardiovascular risk factors and outcomes in early rheumatoid arthritis: a populationbased study. Heart 106:1566

40. Choi HK, Rho Y-H, Zhu Y, Cea-Soriano L, Aviña-Zubieta JA, Zhang Y (2013) The risk of pulmonary embolism and deep vein thrombosis in rheumatoid arthritis: a UK population-based outpatient cohort study. Ann Rheum Dis 72(7):1182-1187

41. Aviña-Zubieta JA, Jansz M, Sayre EC, Choi HK (2017) The risk of deep venous thrombosis and pulmonary embolism in primary Sjögren syndrome: a general population-based study. J Rheumatol 44(8):1184-1189

42. Dalbeth N, Merriman TR, Stamp LK (2016) Gout. Lancet 388(10055):2039-2052. https://doi.org/10.1016/s0140-6736(16) 00346-9
43. Kok VC, Horng J-T, Lin H-L, Chen Y-C, Chen Y-J, Cheng KF (2012) Gout and subsequent increased risk of cardiovascular mortality in non-diabetics aged 50 and above: a population-based cohort study in Taiwan. BMC Cardiovasc Disord 12(1):1-9

44. Luk AJ, Levin GP, Moore EE, Zhou X-H, Kestenbaum BR, Choi HK (2009) Allopurinol and mortality in hyperuricaemic patients. Rheumatology 48(7):804-806

45. Larsen KS, Pottegård A, Lindegaard HM, Hallas J (2016) Effect of allopurinol on cardiovascular outcomes in hyperuricemic patients: a cohort study. Am J Med 129(3):299-306.e292

46. Goicoechea M, de Vinuesa SG, Verdalles U, Ruiz-Caro C, Ampuero J, Rincón A, Arroyo D, Luño J (2010) Effect of allopurinol in chronic kidney disease progression and cardiovascular risk. Clin J Am Soc Nephrol 5(8):1388-1393

47. Kok VC, Horng J-T, Chang W-S, Hong Y-F, Chang T-H (2014) Allopurinol therapy in gout patients does not associate with beneficial cardiovascular outcomes: a population-based matchedcohort study. PLoS ONE 9(6):e99102

48. Corry DB, Eslami P, Yamamoto K, Nyby MD, Makino H, Tuck ML (2008) Uric acid stimulates vascular smooth muscle cell proliferation and oxidative stress via the vascular renin-angiotensin system. J Hypertens 26(2):269-275

49. Mazzali M, Hughes J, Kim Y-G, Jefferson JA, Kang D-H, Gordon KL, Lan HY, Kivlighn S, Johnson RJ (2001) Elevated uric acid increases blood pressure in the rat by a novel crystal-independent mechanism. Hypertension 38(5):1101-1106

50. Andrés M, Bernal JA, Sivera F, Quilis N, Carmona L, Vela P, Pascual E (2017) Cardiovascular risk of patients with gout seen at rheumatology clinics following a structured assessment. Ann Rheum Dis 76(7):1263-1268

51. Gamala M, Jacobs J, Linn-Rasker SP, Nix M, Heggelman B, Pasker-de Jong P, van Laar JM, Klaasen R (2020) Cardiovascular risk in patients with new gout: should we reclassify the risk? Clin Exp Rheumatol 38(3): 533-535

52. Bos MJ, Koudstaal PJ, Hofman A, Witteman JC, Breteler MM (2006) Uric acid is a risk factor for myocardial infarction and stroke: the Rotterdam study. Stroke 37(6):1503-1507

53. Chen L-y, Zhu W-h, Chen Z-w, Dai H-l, Ren J-j, Chen J-h, Chen L-q, Fang L-z (2007) Relationship between hyperuricemia and metabolic syndrome. J Zhejiang Univ Sci B 8(8):593

54. Ali N, Miah R, Hasan M, Barman Z, Mou AD, Hafsa JM, Trisha AD, Hasan A, Islam F (2020) Association between serum uric acid and metabolic syndrome: a cross-sectional study in Bangladeshi adults. Sci Rep 10(1):1-7

55. Tune JD, Goodwill AG, Sassoon DJ, Mather KJ (2017) Cardiovascular consequences of metabolic syndrome. Trans1 Res 183:57-70

Publisher's Note Springer Nature remains neutral with regard to jurisdictional claims in published maps and institutional affiliations. 